\title{
PUMPING OF $\mathrm{H}_{2} \mathrm{O}$ MEGAMASERS
}

\author{
MOSHE ELITZUR \\ Physics $\&$ Astronomy Department \\ University of Kentucky \\ Lexington, KY 40506-0055 \\ USA
}

\begin{abstract}
Strong $\mathrm{H}_{2} \mathrm{O}$ maser emission is detected from active galactic nuclei (AGN) as well as Galactic objects such as star-forming regions and late-type stars. In spite of the widely different luminosities of the different masers, a common pump mechanism seems adequate: neutral collisions at densities of $\sim 10^{8}-10^{10} \mathrm{~cm}^{-3}$ and temperatures of $\sim 250-500 \mathrm{~K}$. The different properties of the various masers can be attributed to geometry. Although disk rotation controls the AGN maser geometry it does not directly determine the dimensions. X-ray radiation and spiral shocks have been suggested as the heating sources of $\mathrm{H}_{2} \mathrm{O}$ megamasers. Both are capable of explaining the observations, and the radiative scenario seems best understood in terms of chance alignment of standard broad-line-region clouds. It is not yet clear whether these different proposals produce distinct maser signatures.
\end{abstract}

\section{Megamasers vs Galactic Masers}

The most luminous Galactic $\mathrm{H}_{2} \mathrm{O}$ maser is $\mathrm{W} 49$, at a distance of $\sim 11 \mathrm{kpc}$ and a typical observed flux of $\sim 10^{4} \mathrm{Jy}$ (Gwinn 1994). At the distance of NGC4258 ( $6 \mathrm{Mpc}$; Miyoshi et al 1995) the flux from W49 is $0.04 \mathrm{Jy}$, comparable to the typical flux level $(\sim 0.1 \mathrm{Jy})$ of that AGN. Flux is proportional to $T_{b} A$, where $T_{b}$ is the source brightness temperature and $A$ its observed area, so an increase by a factor of $\sim 2$ of either observed property of W49- $T_{b}\left(\gtrsim 10^{14} \mathrm{~K}\right)$ or $A$ (implying an increase of only $40 \%$ in observed linear sizes which are $\gtrsim 10^{13} \mathrm{~cm}$ ) - would reproduce the observed flux of NGC4258. Although this seems like a small adjustment, W49 is already at the extreme end of Galactic sources, straining pumping models to their extremes. However, during outbursts, which are not rare (e.g. Liljeström 1993), the flux from W49 rises by factors of $\sim 2-5$. Thus NGC4258 resembles on a day-to-day basis W49 during its outbursts, and can be pumped similarly. The generally accepted pumping mechanism for Galactic $\mathrm{H}_{2} \mathrm{O}$ masers is neutral collisions and the leading explanation for the outbursts is chance alignment in which two maser clumps amplify each other.

\section{Collisional Pumping}

The requirements for collisional pumping of $\mathrm{H}_{2} \mathrm{O}$ masers have been studied extensively and are well understood (Elitzur, Hollenbach \& McKee 1989). Since the model results obey general scaling properties in the relevant regions of parameter space, maser emission is controlled by only three key factors:

- The temperature must exceed $\sim 250 \mathrm{~K}$.

- The density $n=10^{9} n_{9} \mathrm{~cm}^{-3}, \mathrm{H}_{2} \mathrm{O}$ abundance $x\left(\mathrm{H}_{2} \mathrm{O}\right)=10^{4} x_{-4}\left(\mathrm{H}_{2} \mathrm{O}\right)$, source thickness $d=10^{13} d_{13} \mathrm{~cm}$ and line width $\Delta v=10^{5} \Delta v_{5} \mathrm{~cm} \mathrm{~s}^{-1}$ enter only through one scaling parameter $\xi=x_{-4}\left(\mathrm{H}_{2} \mathrm{O}\right) n_{9}^{2} d_{13} / \Delta v_{5}$, the effective emission measure. For efficient maser operation, $\xi$ must be in the range $\sim 1-100$. 
- Planar geometry, providing a long pathlength (in the plane) for the maser photons and a short one (across the plane) for thermal photons. This enables strong maser amplification while avoiding thermalization.

The planar geometry is as important as any other factor. The $\mathrm{H}_{2} \mathrm{O}$ masers in galactic late-type stars and star-forming regions share the same physical parameters and differ only in their geometry. The former occur in winds, the geometry is three-dimensional and the brightness temperatures do not exceed $\sim 10^{11} \mathrm{~K}$. The latter occur behind shocks, the geometry is planar and the brightness temperatures virtually always exceed $\sim 10^{11} \mathrm{~K}$.

Two scenarios were proposed for driving the $\mathrm{H}_{2} \mathrm{O}$ megamaser action in AGN disks: irradiation by the central continuum source (Neufeld, Maloney \& Conger 1994) and, similar to the Galactic masers, spiral shocks (Maoz \& McKee (1997). Both are capable of producing all the pumping requirements. In the radiative heating model the planar geometry arises because the irradiated region has a stratified structure whose layers are perpendicular to the radial direction.

\section{Maser Geometry}

Whatever the underlying mechanism driving the conditions toward maser action, the brightness temperature of a saturated, collisionally pumped planar $\mathrm{H}_{2} \mathrm{O}$ maser is roughly $10^{12} a_{1}^{3} \mathrm{~K}$, where the aspect $a=10 a_{1}=\ell / w$ is the ratio of the maser dimensions along and across the line of sight. At a distance of $D_{\mathrm{Mpc}} \mathrm{Mpc}$, the flux observed from such a maser is

$$
F=0.05 a_{1}\left(\frac{\ell_{15}}{D_{\mathrm{Mpc}}}\right)^{2} \mathrm{Jy}
$$

where $\ell_{15}=\ell / 10^{15} \mathrm{~cm}$. What sets the dimensions of the maser region? Interferometry of NGC 4258 verified the Watson \& Wallin (1994) proposal that the masers delineate directions of maximal velocity coherence in a rotating disk. Further support comes from the Braatz, Wilson \& Henkel (1997) survey which finds preference for megamaser edge-on orientation. If $R$ is the maser's radial distance from the rotation axis and $\Theta=R / D$ is the angular distance then

$$
F=11 \Theta_{\text {mas }}^{2} a_{1}(\ell / R)^{2} \mathrm{Jy}
$$

where $\Theta_{\text {mas }}$ is in milli-arcseconds. For Keplerian rotation $v \propto R^{-1 / 2}$, and velocity coherence, which limits maser amplification to the region whose line of sight velocity obeys $\left|\delta v_{\text {los }}\right| \lesssim \Delta v$, yields

$$
w=3 \times 10^{14} R_{0.1}\left(\frac{10^{3} \Delta v}{v_{\text {los }}}\right) \mathrm{cm}, \quad \ell=8 \times 10^{15} R_{0.1}\left(\frac{10^{3} \Delta v}{v_{\text {los }}}\right)^{1 / 2} \mathrm{~cm}
$$

where $R_{0.1}=R /(0.1 \mathrm{pc})$. The $\mathrm{H}_{2} \mathrm{O}$ maser flux of the full velocity-coherent region is thus

$$
F=.024 \Theta_{\operatorname{mas}}^{2}\left(\frac{10^{3} \Delta v}{v_{\mathrm{los}}}\right)^{1 / 2} \mathrm{Jy}
$$

In NGC 4258 , where $\Theta_{\text {mas }} \approx 4-8$ and $\Delta v / v_{\text {los }} \approx 10^{-3}$, this result gives $F \approx 0.4-1.5 \mathrm{Jy}$, up to almost an order of magnitude more than the typical flux level of the maser features in this source. The implication is that only a fraction of the potentially observable volume actually contributes to the maser emission. The situation is similar in other AGNs whose high-velocity maser features have been imaged. So the flux levels imply that the dimensions of the emission regions must be smaller than those of the full velocity-coherence "box". Moreover, since for Keplerian rotation $\Theta \propto v^{-2}$, the last relation also gives

$$
F \propto \frac{1}{v_{\mathrm{los}}^{4.5}}
$$

for a constant linewidth $\Delta v$. Such a spectral shape has never been observed thus far. In the first interferometric mapping of NGC 4258 on 1994 April 26, the flux of the high-velocity features did exhibit a trend of decreasing with $v_{\text {los }}$ (see Fig. 1 in Miyoshi et al. 1995), but the decline was much milder than $v_{\text {los }}^{-4.5}$. In subsequent mapping on 1995 January 8 the highest-velocity peaks rose sharply 
so that the flux was actually increasing with $v_{\mathrm{los}}$ toward the high end of the spectrum (Herrnstein et al. 1997), the opposite of the trend predicted by velocity coherence. This spectral shape remained virtually unchanged in the latest reported mapping on 1995 May 28. Hence, independent of the normalization of the pump estimates, one concludes that the maser sizes do not sample the disk velocity field, i.e., line-of-sight velocity coherence DOES NOT directly determine the size of the maser region! This presents a puzzle. If the maser is smaller than the velocity-coherent region, how are the directions of maximal coherence picked?

Modeling attempts must resolve these somewhat conflicting requirements: Although velocity coherence in a rotating disk clearly does control the observed pattern of maser emission, it does not directly set the maser dimensions. Another length scale must be introduced by some additional physical ingredient. In the model of Maoz \& McKee (1997; see also Maoz 1997), this additional scale can be set by the radius of curvature of spiral shocks in a rotating disk. The central irradiation scenario, too, can naturally meet these requirements if the maser radiation origiriates in discrete clouds (Kartje, Königl \& Elitzur 1997). The basic maser scale is then set by the size of the illuminated part of the cloud where appropriate temperature and $\mathrm{H}_{2} \mathrm{O}$ abundance are maintained. These regions are similar in all relevant properties to individual maser spots in Galactic star-forming regions. The observed flux levels are reached through chance alignment along the line of sight of two maser clouds amplifying each other, and are controlled by the cloud separation. Since the clouds have a Keplerian disk-like distribution, the preference for edge-on orientations, which have the best chance for alignment, is preserved. An interesting result is that the observed flux is largely determined by cloud separation and is almost independent of the specific properties of the individual clouds (provided, of course, they fall in the maser-producing regime). If we denote by $a_{*}=R_{c} / w_{c}$ the ratio of the cloud radius to the width of its maser-producing region $\left(1 / a_{*}\right.$ is the fractional size of the region where appropriate temperature and $\mathrm{H}_{2} \mathrm{O}$ abundance are maintained), then the flux from overlapping clouds separated by distance $s=10^{16} s_{16} \mathrm{~cm}$ is

$$
F \approx 5.4 \frac{s_{16}^{2} a_{*, 1}^{1 / 2}}{D_{\mathrm{Mpc}}^{2}} \quad \mathrm{Jy}
$$

where $a_{*}=10 a_{*, 1}$. Flux comparable to observed is produced for separations close to the maximum allowed by velocity coherence. The dependence on individual cloud properties enters only weakly through the aspect ratio $a_{*}$.

The flux dependence on chance alignment within the velocity-coherent region provides a natural explanation for the variations of overall spectral shape and the strong (up to a factor of $\sim 3$ ) variations in the flux measured from some of the high-velocity features in NGC 4258 on time scales of a few months. A similar behavior was also noted in NRO observations of NGC 5793 (Hagiwara et al. 1997). Such variability can be readily interpreted in terms of poloidal motion at a speed of a few $\mathrm{km} \mathrm{s}^{-1}$ of individual clouds whose emission regions move into (or out of) alignment with each other along the line of sight to the observer, similar to outbursts in Galactic $\mathrm{H}_{2} \mathrm{O}$ masers.

In this scenario, the dense clouds located within the dusty molecular torus that is believed to surround the broad emission-line region of Seyfert galaxies provide a natural site for AGN $\mathrm{H}_{2} \mathrm{O}$ megamaser activity. In fact, the requisite cloud number densities and their internal densities, $\sim 10^{8}$. $10^{10} \mathrm{~cm}^{-3}$, are consistent with the characteristic values of the familiar broad emission-line clouds. Dust shielding of the central continuum may play a key role in determining the location and extent of the emission region. Shielding by a dusty intercloud medium with a column density $\gtrsim 10^{23}$ $\mathrm{cm}^{-2}$ is crucial for the survival of molecules in the intense ambient radiation field at the observed locations of the masers. Since the maser-shielding column is directly related in this picture to the equivalent X-ray-absorbing hydrogen column density, this model naturally explains the inferred correlation between the probability of detecting $\mathrm{H}_{2} \mathrm{O}$ maser emission in a Seyfert galaxy and the magnitude of its X-ray-absorbing column (Braatz, Wilson \& Henkel 1997). The dust sublimation radius provides a lower limit on the inner radius of the maser emission region, which agrees well with observed values. The outer radius of this region may be associated with the distance where the shielding column becomes so large that the temperature in the masing clouds drops below the threshold $(\sim 250 \mathrm{~K})$ for efficient collisional pumping. 


\section{Conclusions}

Two basic scenarios have been proposed for driving the $\mathrm{H}_{2} \mathrm{O}$ masers in AGNs - central X-ray radiative heating and spiral shocks. Both are capable of producing the physical parameters necessary for maser action as well as sizes smaller then line-of-sight velocity coherence combined with preference for edge-on disks. There are no clear emission diagnostics that distinguish the two scenarios and at this time both seem viable candidates.

\section{References}

Braatz, J. A., Wilson, A. S., \& Henkel, C. 1997, ApJS, 110, 321

Elitzur, M., Hollenbach, D. J., \& McKee, C. F. 1989, ApJ, 346, 983

Gwinn, C.R. 1994, Ap.J 429, 241

Hagiwara, Y., Kohno, K., Kawabe, R., \& Nakai, N. 1997, PASJ, 49, 171

Herrnstein, J. R., et al. 1997, in ASP Conf. Ser., The Physics of LINERS in View of Recent Observations, ed. M. Eracleous, A. P. Koratkar, L. C. Ho,\& C. Leitherer (San Francisco: ASP), in press

Kartje, J. F., \& Königl, \& Elitzur, M. 1997, Ap.J (submitted)

Liljeström, T. 1993, in Astrophysical Masers, eds. A.W. Clegg \& G.E. Nedoluha (Springer-Verlag), p. 291

Maoz, E., 1997, these proceedings

Maoz, E., \& McKee, C. F. 1997, ApJ, in press

Miyoshi, M., et al. 1995, Nature, 373, 127

Neufeld, D. A., Maloney, P. R., \& Conger, S. 1994, ApJ, 436, L127

Watson, W. D., \& Wallin, B. K. 1994, ApJ, 432, L35 